OPEN ACCESS

Edited by:

Huaxi Yi,

Ocean University of China, China

Reviewed by:

Fei Liu,

Northeast Agricultural University,

China

Jiachao Zhang,

Hainan University, China

*Correspondence:

Fengwei Tian

fwtian@jiangnan.edu.cn

Specialty section:

This article was submitted to

Nutritional Immunology,

a section of the journal

Frontiers in Immunology

Received: 04 July 2021

Accepted: 22 July 2021

Published: 10 August 2021

Citation:

Yu L, Zhang L, Duan H, Zhao R,

Xiao Y, Guo M, Zhao J, Zhang $H$, Chen $W$ and Tian F (2021) The Protection of Lactiplantibacillus plantarum CCFM8661 Against Benzopyrene-Induced Toxicity via Regulation of the Gut Microbiota.

Front. Immunol. 12:736129. doi: 10.3389/fimmu.2021.736129

\section{The Protection of Lactiplantibacillus plantarum CCFM8661 Against Benzopyrene-Induced Toxicity via Regulation of the Gut Microbiota}

\author{
Leilei Yu ${ }^{1,2,3}$, Lingyu Zhang ${ }^{1,2}$, Hui Duan ${ }^{1,2}$, Ruohan Zhao ${ }^{1,2}$, Yue Xiao $^{1,2}$, Min Guo $^{1,2}$, \\ Jianxin Zhao ${ }^{1,2,3}$, Hao Zhang ${ }^{1,2,3}$, Wei Chen ${ }^{1,2,3}$ and Fengwei Tian ${ }^{1,2,3 *}$ \\ ${ }^{1}$ State Key Laboratory of Food Science and Technology, Jiangnan University, Wuxi, China, ${ }^{2}$ School of Food Science and \\ Technology, Jiangnan University, Wuxi, China, ${ }^{3}$ National Engineering Research Center for Functional Food, Jiangnan \\ University, Wuxi, China
}

The present study evaluated the protection of Lactiplantibacillus plantarum CCFM8661, a candidate probiotic with excellent benzopyrene (B[a]P)-binding capacity in vitro, against $\mathrm{B}$ [a]P-induced toxicity in the colon and brain of mice. Mice that received B[a]P alone served as the model group. Each mouse in the $L$. plantarum treatment groups were administered $2 \times 10^{9}$ colony forming unit (CFU) of $L$. plantarum strains once daily, followed by an oral dose of B[a]P at $50 \mathrm{mg} / \mathrm{kg}$ body weight. Behavior, biochemical indicators in the colon and brain tissue, and the gut microbiota composition and short-chain fatty acid (SCFA) levels in the gut were investigated. Compared to the treatment in the model group, CCFM8661 treatment effectively reduced oxidative stress in the brain, improved behavioral performance, increased intestinal barrier integrity, and alleviated histopathological changes in mice. Moreover, CCFM8661 increased the gut microbiota diversity and abundance of Ruminococcus and Lachnospiraceae and reduced the abundance of pro-inflammatory Turicibacter spp. Additionally, the production of SCFAs was significantly increased by L. plantarum CCFM8661. Our results suggest that CCFM8661 is effective against acute B[a]P-induced toxicity in mice and that it can be considered as an effective and easy dietary intervention against B[a]P toxicity.

Keywords: probiotic, Lactiplantibacillus plantarum, benzopyrene, gut microbiota, SCFAs, gut barrier, behavioral performance

\section{INTRODUCTION}

Benzopyrene $(\mathrm{B}[\alpha] \mathrm{P})$ is a kind of polycyclic aromatic hydrocarbon that is categorized as a group I carcinogen by the International Agency for Research on Cancer $(1,2)$. Contaminated food is an important source of human exposure to $\mathrm{B}[\alpha] \mathrm{P}$, which is up to $125 \mathrm{ng}$ per day for each person $(2,3)$. $\mathrm{B}[\alpha] \mathrm{P}$ would be produced during food processing, such as smoking, grilling, frying. $\mathrm{B}[\alpha] \mathrm{P}-$ contaminated foods include vegetables $(13 \mathrm{ng} / \mathrm{kg})$, cereals $(262 \mathrm{ng} / \mathrm{kg})$, smoked fish $(800-13900$ $\mathrm{ng} / \mathrm{kg}$ ), and dairy products (11-78 ng/kg) (4). After entering into the host's body, $\mathrm{B}[\alpha] \mathrm{P}$ would bind to the aryl-hydrocarbon receptor and be activated by cytochrome P-4501A1 (CYP1A1) to produce 
immense size DNA adducts and $\operatorname{ROS}(2,5)$, which would inactivate tumor suppressor genes or activate oncogenes, resulting in DNA damage, mutation, and cancer $(6,7)$.

$\mathrm{B}[\alpha] \mathrm{P}$ is neurotoxic and it affects the levels of 5hydroxytryptamine, 5-hydroxyindoleacetic acid, and target protein kinase $C(8)$, leading to neurotoxicity and behavioral disturbances $(6,9)$. More seriously, the neurotoxicity of $\mathrm{B}[\alpha] \mathrm{P}$ has a genetic effect (10). However, the effects of $\mathrm{B}[\alpha] \mathrm{P}$ on the gut have not been thoroughly studied so far. The intestinal tract is the first vital barrier against oral $\mathrm{B}[\alpha] \mathrm{P}$ exposure. Therefore, it is necessary to investigate the negative effects of $\mathrm{B}[\alpha] \mathrm{P}$ on the gut, especially the gut microbiota, gut barrier and intestinal histopathology. Previous researches have reported the harmful effects of $\mathrm{B}[\alpha] \mathrm{P}$ on the intestinal barrier, such as changes in the expression levels of tight junction (TJ) proteins $(11,12)$. Ribiere et al. demonstrated that $\mathrm{B}[\alpha] \mathrm{P}$ exposure dramatically changed the gut microbiota composition, thereby causing a proinflammatory intestinal environment and leading to moderate inflammation in ileum and colon of mice (13). Moreover, the important roles of intestine microbiota and SCFAs in regulating host health have aroused the increasing concern (14-16). For example, gut microbiota-generated SCFAs promote metabolism via gut-brain axis, thereby reducing body weight, adiposity, and blood glucose (15).

Lactic acid bacteria (LAB), a group of safe gram-positive microorganisms, are widely used in various fermented food (17). $\mathrm{LAB}$ are also the important components of the human gut microbiota with various physiological function, including balance of immune cell, regulation of metabolites and gut microbiota. Lactiplantibacillus plantarum is a super vital LAB that is capable of binding or sequestering various carcinogens in vitro and in vivo and is a safe and cost-effective intervention way (18-20). Apart from its capacity to bind carcinogens, L. plantarum regulates the gut microbiota and has antioxidative properties, which may be important for the alleviation of $\mathrm{B}[\alpha] \mathrm{P}$ toxicity. Therefore, the aim of this study was to select a novel probiotic strain with excellent $\mathrm{B}[\alpha] \mathrm{P}$-binding ability from the $23 \mathrm{~L}$. plantarum strains tested and evaluate its protective effects on the colons and brains of mice exposed to $\mathrm{B}[\alpha] \mathrm{P}$, especially the impacts on the intestinal microbiota composition and intestinal barrier function. Moreover, possible protective mechanisms against $B[\alpha] P$ toxicity are proposed.

\section{MATERIAL AND METHODS}

\section{Bacterial Strains and Culture}

Twenty-three Lactiplantibacillus plantarum strains, including L. plantarum CCFM571, CCFM595, CCFM8610, CCFM438, CCFM726, CCFM408, CCFM8661, CCFM634, CCFM175, CCFM242, CCFM361, CCFM259, CCFM382, Lp45, FJSWX145, DYNDL58M4, PS3-9, FFJND7-L5, HY9-10, M2-05-R02, FJSZJ4-L5, 4L-4 and VNMWLT1M12, were obtained from Research Center of Food Biotechnology in Jiangnan University (Wuxi, China). The cultivation of all L. plantarum strains was in MRS broth (Hopebio, Qingdao, China) at $37^{\circ} \mathrm{C}$ for $18 \mathrm{~h}$.

\section{Determination of the $B[\alpha]$ P-Binding Capacity of $L$. plantarum Strains In Vitro}

The $\mathrm{B}[\alpha] \mathrm{P}$-binding ability of the $23 \mathrm{~L}$. plantarum strains was estimated as previously described (21). The cultured biomass was centrifuged at 5,000 $\times g$ for $15 \mathrm{~min}$ and washed twice with ultrapure water to acquire cell pellets. The cell pellets were re-suspended in the ultrapure water containing $10 \mu \mathrm{g} / \mathrm{mL} \mathrm{B}[\alpha] \mathrm{P}$ (Sigma-Aldrich, St Louis, MO, USA). The suspension was centrifuged after incubation for $2 \mathrm{~h}$ at $150 \mathrm{rpm}$ and $37^{\circ} \mathrm{C}$, and the residual $\mathrm{B}[\alpha] \mathrm{P}$ concentration in the supernatant was analyzed. $\mathrm{B}[\alpha] \mathrm{P}$ levels were measured using a high performance liquid chromatography (HPLC) equipped with a Waters Atlantis C18 reverse-phase column $\left(4.6 \times 250 \mathrm{~mm} \times 5 \mu \mathrm{m}, 30^{\circ} \mathrm{C}\right.$; Waters Corporation, Milford, MA, USA). The mobile phase was acetonitrile:water (88:12). The injection volume was $20 \mu \mathrm{L}$, with a flow rate of $1 \mathrm{~mL} / \mathrm{min}$, and the fluorescence detection wavelength was $406 \mathrm{~nm}$.

The $\mathrm{B}[\alpha] \mathrm{P}$-binding abilities of the Lactiplantibacillus strains are expressed as the $\mathrm{B}[\alpha] \mathrm{P}$ removal rate, which was calculated as follows:

Removal rate $(\%)=\left[\left(\mathrm{C}_{\mathrm{i}}-\mathrm{C}_{\mathrm{r}}\right) / \mathrm{C}_{\mathrm{i}}\right] \times 100 \%, \mathrm{C}_{\mathrm{i}}$ and $\mathrm{C}_{\mathrm{r}}$ are the initial and residual $\mathrm{B}[\alpha] \mathrm{P}$ level, respectively.

\section{Animal Experimental Procedure}

Male adult BALB/c mice (8-week-old) were purchased from Slack limited company (Shanghai, China). Mice were kept in cages at a constant temperature $\left(22^{\circ} \mathrm{C} \pm 1^{\circ} \mathrm{C}\right)$ and humidity $(55 \% \pm 10 \%)$ under a $12-\mathrm{h} / 12-\mathrm{h}$ light/dark cycle and had free access to food and water. All procedures and protocols of mice experiments were performed according to the guidelines of the Animal Care and Use Committee and the Ethics Committee of Jiangnan University (JN.No20190915b0481210).

The mice were divided into four groups and allowed to acclimatize to their environment for 1 week. The experimental schedule is shown in Table 1. Group 1 (control group) was administered skim milk and corn oil without $\mathrm{B}[\alpha] \mathrm{P}$. Group 2 (model group) was administered $\mathrm{B}[\alpha] \mathrm{P}$ dissolved in corn oil at a dose of $50 \mathrm{mg} / \mathrm{kg}$ b.w. Groups 3 and 4 (CCFM8661 and CCFM382 groups, respectively) were administered $2 \times 10^{9} \mathrm{CFU}$ of L. plantarum strains CCFM8661 and CCFM382, respectively, and $50 \mathrm{mg} / \mathrm{kg}$ b.w. of $\mathrm{B}[\alpha] \mathrm{P}$. All treatments were administered via oral gavage for 5 weeks. Mice were fasted for $12 \mathrm{~h}$ before sacrifice. Blood samples were collected and centrifuged at 3,000 $\times$ $g$ for 15 min to obtain the serum, which was used for biochemical analysis. Colon and brain tissues were immediately washed with

TABLE 1 | Animal experimental protocol.

\begin{tabular}{ll}
\hline Group $\mathbf{( n = 8 )}$ & \multicolumn{1}{c}{ Treatment (5 weeks) } \\
\hline Control & $\mathrm{SM}+\mathrm{CO}$ \\
Model & $\mathrm{SM}+\mathrm{B}[\alpha] \mathrm{P}$ \\
CCFM8661 & L. plantarum CCFM8661+B $[\alpha] \mathrm{P}$ \\
CCFM382 & L. plantarum CCFM382+B $\alpha] \mathrm{P}$
\end{tabular}

$\mathrm{CO}=0.2 \mathrm{~mL}$ corn oil; $S M=0.2 \mathrm{~mL}$ skim milk; $B[\alpha] P=0.2 \mathrm{~mL}$ corn oil containing $50 \mathrm{mg} / \mathrm{kg}$ body weight of $B[\alpha] P$; CCFM8661 $=0.2 \mathrm{~mL}$ skim milk containing $2 \times 10^{9} \mathrm{CFU}$ of $\mathrm{L}$. plantarum CCFM8661; CCFM382 $=0.2 \mathrm{~mL}$ skim milk containing $2 \times 10^{9} \mathrm{CFU}$ of $\mathrm{L}$. plantarum CCFM382. Animals received corn oil, skim milk, B[A]P, and L. plantarum strains via gavage. 
$0.9 \%$ saline and separated into two parts: one part was stored at $-80^{\circ} \mathrm{C}$ for subsequent measurements, and the other part was fixed with $4 \%$ formalin for histopathological analysis.

\section{Open-Field Test}

The test was performed as previously described with minor modifications $(22,23)$. The size of apparatus used for the openfield test was $50 \mathrm{~cm} \times 50 \mathrm{~cm}$ square with four white walls. The edge region is a $15 \mathrm{~cm}$ area near the walls and the rest of the field was the central area. Each mouse can move freely within the apparatus for $15 \mathrm{~min}$. A camera was used to record their movements, and the data were analyzed using EthoVision (Noldus, Wageningen, Netherlands). The apparatus was cleaned with $75 \%$ ethanol after each test to eliminate any possible odor cues. The total distance traveled and the times spent in the center and the edge regions were calculated to measure anxiety-like behavior.

\section{Determination of 3-OH $\mathrm{B}[\alpha] \mathrm{P}$ Levels in Feces}

The mouse feces were mixed with acetonitrile (1:2) by vortex oscillation. After centrifugation at $10000 \mathrm{rpm}$ for $10 \mathrm{~min}$, the 3$\mathrm{OH} B[\alpha] \mathrm{P}$ levels in supernatant was analyzed by HPLCfluorescence detection (24). The mobile phase was methanol: water (97:3, pH4.5). The injection volume was $20 \mu \mathrm{L}$, with a flow rate of $0.5 \mathrm{~mL} / \mathrm{min}$, and the fluorescence excitation and emission wavelength were 365 and $450 \mathrm{~nm}$, respectively.

\section{Determination of Oxidative Stress-Related Parameters in the Brain}

The MDA level and SOD activity were measured using ELISA kit according to the operating instructions of the manufacturer (Jiancheng Bioengineering, Nanjing, China).

\section{RT-qPCR Analysis}

Colon and brain tissue $(0.1 \mathrm{~g})$ samples were lysed in TRIzol reagent (Ambion, USA) for RNA extraction. cDNA was synthesized using the RevertAid (Thermo Fisher Scientific, Waltham, MA, USA). Gene expression levels were determined using validated primers for Gapdh, Zo-1, Occludin, Claudin-1, CYP1A1, Bax, Bcl-2, and p53 (Table 2) and iTaq Univeral (Bio-Rad, Hercules, CA, USA) on an RT-qPCR system (BioRad-CFX384) (25). The PCR program comprised initial denaturation at $95^{\circ} \mathrm{C}$ for $2 \mathrm{~min}$, followed by 40 cycles of $95^{\circ} \mathrm{C}$ for $30 \mathrm{~s}, 60^{\circ} \mathrm{C}$ for $30 \mathrm{~s}$, and $72^{\circ} \mathrm{C}$ for $30 \mathrm{~s}$, and finally, $72^{\circ} \mathrm{C}$ for $5 \mathrm{~min}$. Relative quantification of these target gene expression levels was performed after normalization to Gapdh gene expression levels using the $2^{-\Delta \Delta \mathrm{Ct}}$ method.

\section{Histopathological Analysis}

Colon tissue was fixed in $10 \%$ formalin saline for $24 \mathrm{~h}$ and then embedded in paraffin. The paraffin was sliced into 5 - $\mu$ m-thick sections. After sectioning, the tissue samples were stained with H\&E (26).

\section{Analysis of the Gut Microbiota in Feces}

Total DNA in feces was extracted using the FastDNA Spin Kit (MP Biomedicals, Santa Ana, CA, USA). The V3-V4 region of the $16 \mathrm{~S}$ rRNA gene was amplified using 341F/806R primers.
TABLE 2 | Primer sequences used for RT-qPCR.

\begin{tabular}{|c|c|c|}
\hline \multirow{2}{*}{$\begin{array}{l}\text { Primer } \\
\text { GAPDH }\end{array}$} & \multicolumn{2}{|c|}{ Sequence (5'-3') } \\
\hline & Forward (F) & TGCACCACCAACTGCTTAG \\
\hline & Reverse (R) & GATGCAGGGATGATGTTC \\
\hline \multirow[t]{2}{*}{ ZO-1 } & $\mathrm{F}$ & СТTCTCTTGCTGGCCCTAAAC \\
\hline & $\mathrm{R}$ & TGGCTTCACTTGAGGTITCTG \\
\hline \multirow[t]{2}{*}{ Occludin } & $\mathrm{F}$ & CACACTTGCTTGGGACAGAG \\
\hline & $\mathrm{R}$ & TAGCCATAGCCTCCATAGCC \\
\hline \multirow[t]{2}{*}{ Claudin-1 } & $\mathrm{F}$ & GATGTGGATGGCTGTCATTG \\
\hline & $\mathrm{R}$ & CCTGGCCAAATTCATACCTG \\
\hline \multirow[t]{2}{*}{ CYP1A1 } & $\mathrm{F}$ & CCTCATGTACCTGGTAACCA \\
\hline & $\mathrm{R}$ & AAGGATGAATGCCGGAAGGT \\
\hline \multirow[t]{2}{*}{ Bax } & $\mathrm{F}$ & CTACAGGGTTTCATCCAG \\
\hline & $\mathrm{R}$ & CCAGTTCATCTCCAATTCG \\
\hline \multirow[t]{2}{*}{$\mathrm{Bcl}-2$} & $\mathrm{~F}$ & GTGGATGACTGAGTACCT \\
\hline & $\mathrm{R}$ & CCAGGAGAAATCAAACAGAG \\
\hline \multirow[t]{2}{*}{ P53 } & $\mathrm{F}$ & GTATITCACCCTCAAGATCC \\
\hline & $\mathrm{R}$ & TGGGCATCCTITAACTCTA \\
\hline
\end{tabular}

The library was built and sequenced on an Illumina MiSeq PE300 platform.

\section{Determination of SCFA Levels}

Fecal samples (50mg) were dispersed in $50 \mu \mathrm{L}$ of saturated $\mathrm{NaCl}$ solution, acidified with $5 \%(\mathrm{v} / \mathrm{v}) \mathrm{H}_{2} \mathrm{SO}_{4}$, and SCFAs were extracted with $1 \mathrm{~mL}$ of diethyl ether. SCFA levels were tested by GC-MS (27).

\section{Statistical Analysis}

Statistical analyses were performed using Prism version 7 (GraphPad, San Diego, CA, USA). Significant differences were evaluated using a one-way analysis of variance. Microbiotarelated analyses, including alpha diversity and biodiversity richness, were assessed with the QIIME (version 1.17) and $\mathrm{R}$ (version 3.5.0) software.

\section{RESULTS}

\section{B[a]P-Binding Abilities}

The $\mathrm{B}[\mathrm{a}] \mathrm{P}$-binding abilities of the $23 \mathrm{~L}$. plantarum strains are presented in Figure 1. The $\mathrm{B}[\mathrm{a}] \mathrm{P}$-binding abilities were significantly different among the different $L$. plantarum strains. L. plantarum CCFM8661 had the highest B[a]P removal capacity, with a removal rate of $60.9 \%$; thus, this strain was selected as the target strain for subsequent animal experiments. CCFM382 had the lowest removal rate of only $1.6 \%$; thus, it was selected as the negative reference strain. The protection of these two L. plantarum strains against $\mathrm{B}[\alpha] \mathrm{P}$-induced toxicity in the gut and the brain were compared.

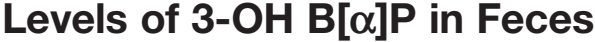

The levels of 3-OH B $[\alpha] \mathrm{P}$ in the feces of mice significantly increased after $\mathrm{B}[\mathrm{a}] \mathrm{P}$ treatment, which was up to $0.27 \mu \mathrm{g} / \mathrm{g}$ (Figure $2 ; P<0.05$ ). The effects were significantly reversed by oral administration of L. plantarum CCFM8661 $(P<0.05)$, but not L. plantarum CCFM382 $(P>0.05)$. The $3-\mathrm{OH} \mathrm{B}[\alpha] \mathrm{P}$ level in the CCFM8661 and CCFM382 groups were $0.14 \mu \mathrm{g} / \mathrm{g}$, and $0.21 \mu \mathrm{g} / \mathrm{g}$, respectively. 


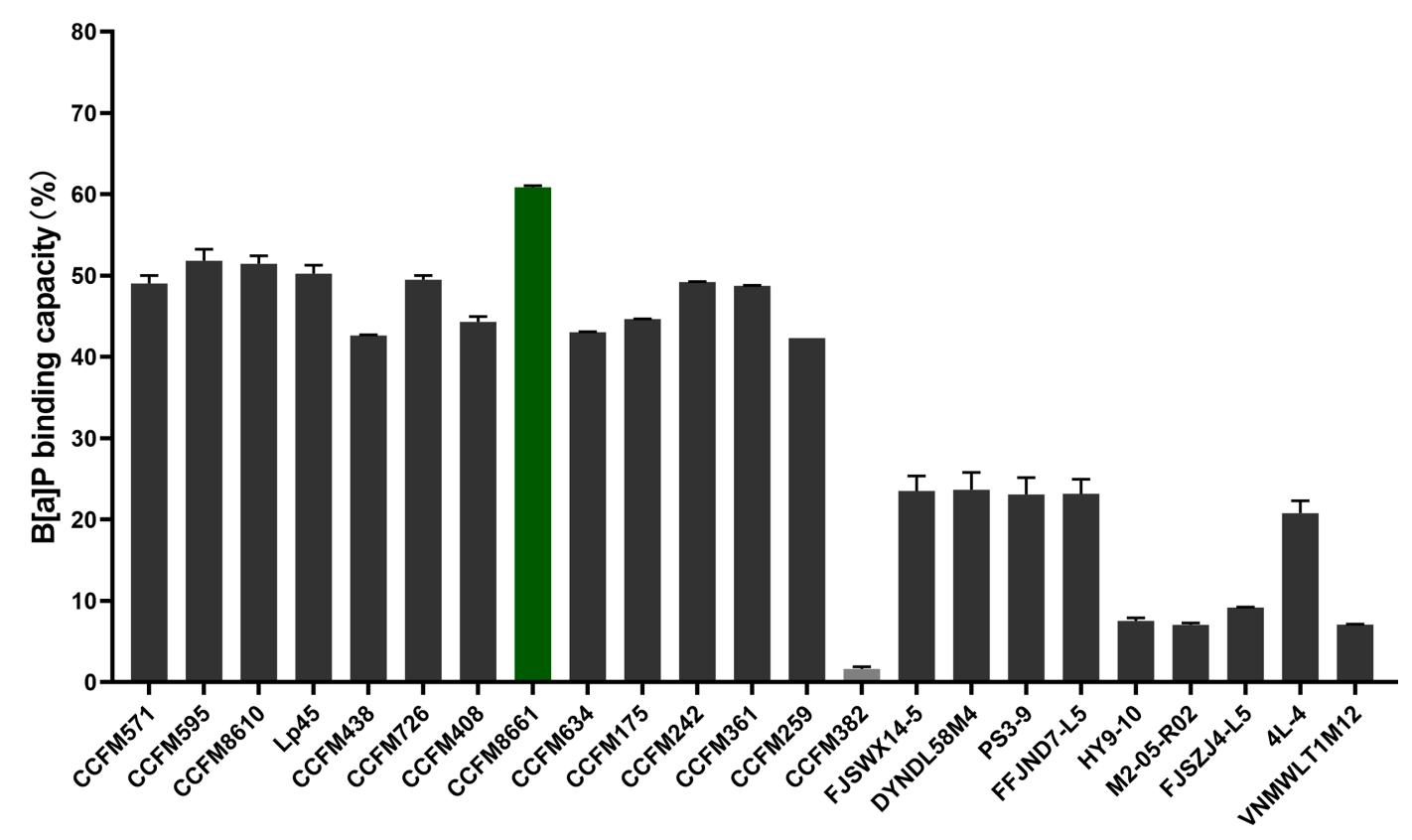

FIGURE 1 | B[ $\alpha]$ P-binding capacity of $L$. plantarum strains.

\section{Open-Field Test}

The open-field test was used to evaluate the spatial cognitive ability of the experimental animals. The distance moved was used to represent autonomous activity ability, and the time spent in the central area was used to reflect their spatial cognition ability in a new environment. Mice with poor cognitive ability would quickly leave the central area and move along the periphery, thereby spending less exploration time in the central area. As shown in Figure 3, B $[\alpha]$ P-treated mice traveled a shorter total distance and spent less time in the center than mice in the control group $(P<0.05)$. However, mice in the CCFM8661 and CCFM382 group traveled a longer distance and spent more time in the central zone than those in the model group $(P<0.05)$. Importantly, L. plantarum CCFM8661 had a more significant increase on these two parameters than CCFM382. These results suggested that anxiety-like behavior caused by $\mathrm{B}[\alpha] \mathrm{P}$ can be better reversed by CCFM8661 supplementation.

\section{Oxidative Stress- and Tumor-Related Parameters in the Brain}

Malondialdehyde (MDA) levels were dramatically higher in the model group than those in the control group (Figure $\mathbf{4 B}$, $P<0.05)$. Of the two $L$. plantarum intervention groups, only $L$. plantarum CCFM8661 sharply decreased the MDA levels $(P<0.05)$. Superoxide dismutase activity was not dramatically different between the groups (Figure 4A).

The expression levels of $B c l-2$ and $p 53$ in the brain increased dramatically in the model group, but L. plantarum CCFM8661 dramatically reduced the expression levels of these genes
(Figures 4D, E; $P<0.05)$. Although the decrease expression of p53 in CCFM382 group was also observed, the difference was not significant $(P>0.05)$. In addition, the Bax expression level in the brain tissue of the control, model, and two L. plantarumintervention groups were no significant differences (Figure 4C).

\section{The mRNA Expression of TJ Proteins and CYP1A1 in the Colon}

The mRNA expression levels of $\mathrm{Zo}-1$ and occludin in the colon were dramatically lower in $\mathrm{B}[\alpha] \mathrm{P}$-exposed mice than in control mice (Figure 5A; $P<0.05$ ). Oral administration of CCFM8661 and CCFM382 significantly increased occludin expression $(P<0.05)$. The mRNA expression levels of claudin-1 were increased in the model group $(P<0.05)$ but were not significantly affected by $L$. plantarum intervention $(P>0.05)$.

Levels of the B[a]P-metabolizing enzyme CYP1A1, a member of the P450 enzyme family, were increased about six-fold after B [a]P treatment (Figure 5A; $P<0.05$ ), and L. plantarum CCFM8661, rather than L. plantarum CCFM382, significantly reduced its expression $(P<0.05)$, thus alleviating the damage to the colon caused by $\mathrm{B}[\mathrm{a}] \mathrm{P}$.

\section{Histopathological Changes in the Colon}

Colonic histopathology was normal in mice in the control group (Figure 5B). However, $\mathrm{B}[\alpha] \mathrm{P}$ treatment led to serious injury to the colon, including crypt destruction, inflammatory cell infiltration, and severe ulceration. Colonic ulcers were reversed after L. plantarum CCFM382 administration, but there was still moderate inflammatory cell infiltration. Treatment with $L$. plantarum CCFM8661 significantly alleviated the colonic histopathological lesions to almost normal levels. Therefore, 


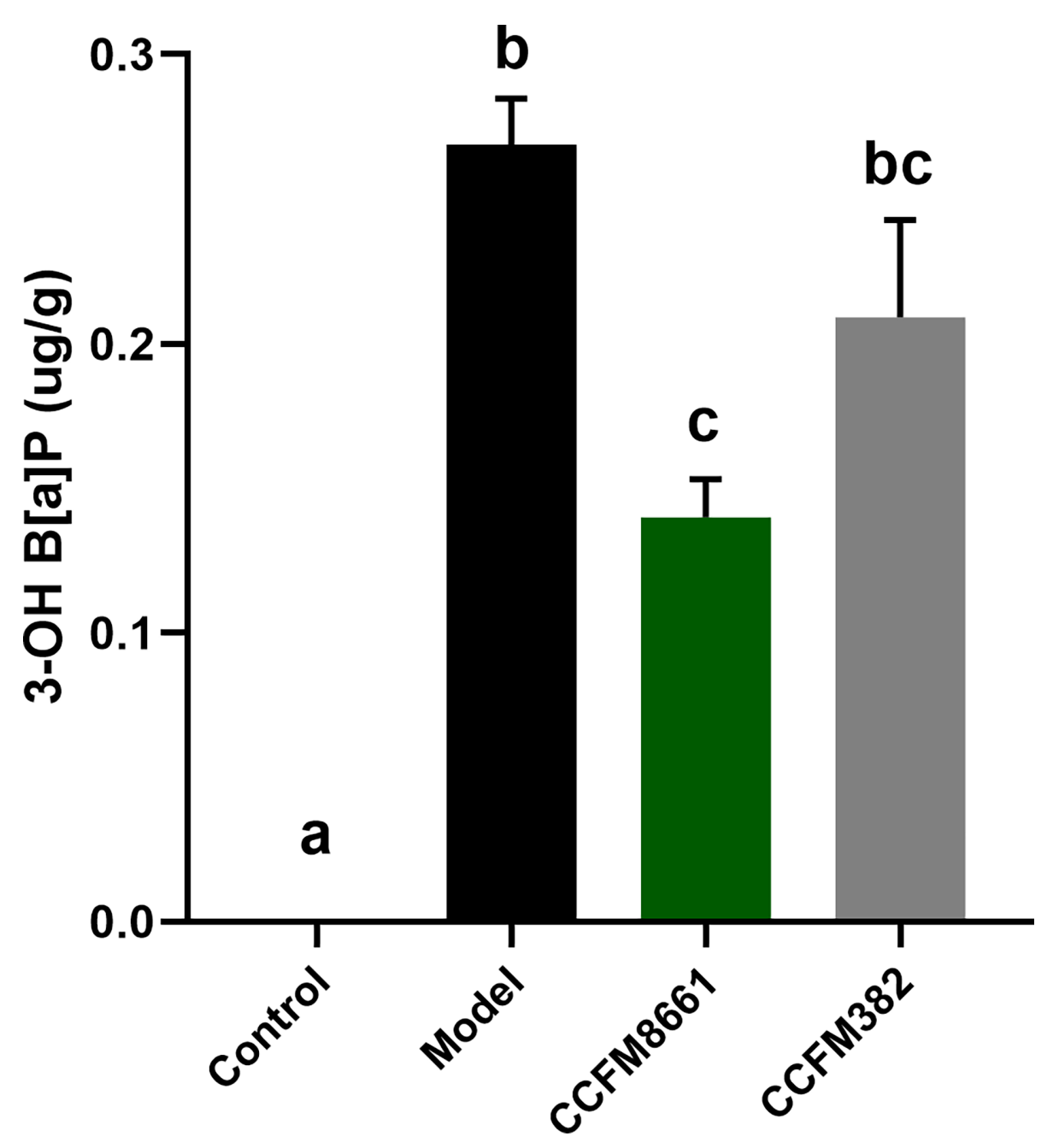

FIGURE 2 | Effect of $L$. plantarum CCFM8661 on 3-OH B[ $\alpha]$ P levels in feces. The different letters indicate significant difference between groups $(P<0.05)$.
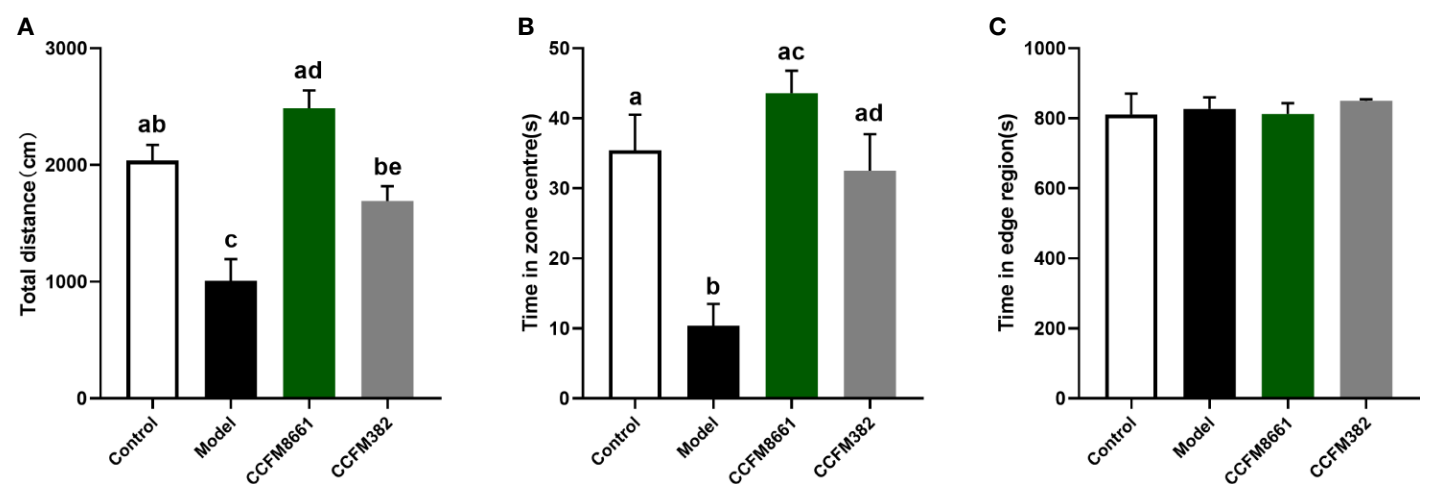

FIGURE 3 | Effects of $L$. plantarum CCFM8661 on B[ $\alpha]$ P-induced behavioral changes. (A) The total distance, (B) The time spend in zone centres, (C) The time spend in edge region. The different letters indicate significant difference between groups $(P<0.05)$. 

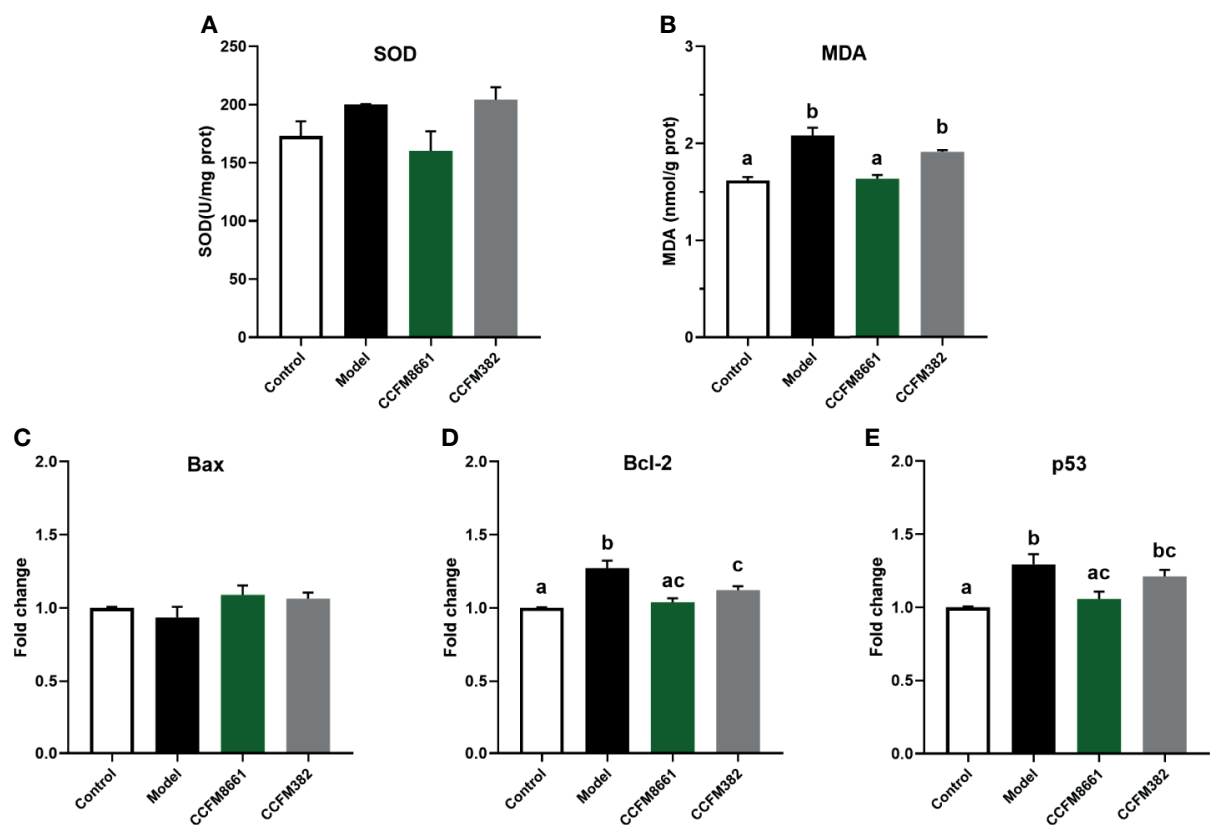

FIGURE 4 | Effect of L. plantarum CCFM8661 on oxidative stress- and tumor-related parameters in the brain. (A) SOD activity, (B) MDA level, (C-E) mRNA expression levels of apoptosis-related genes in brain. The different letters indicate significant difference between groups $(P<0.05)$.
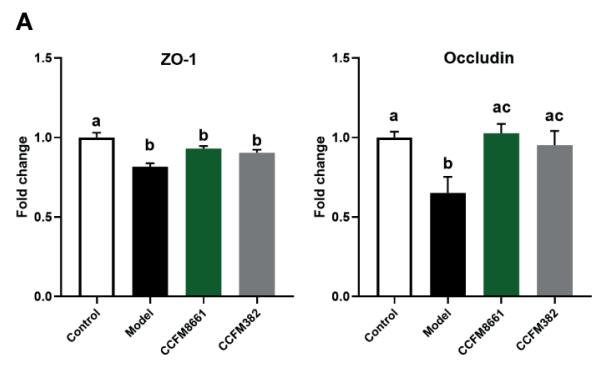

B
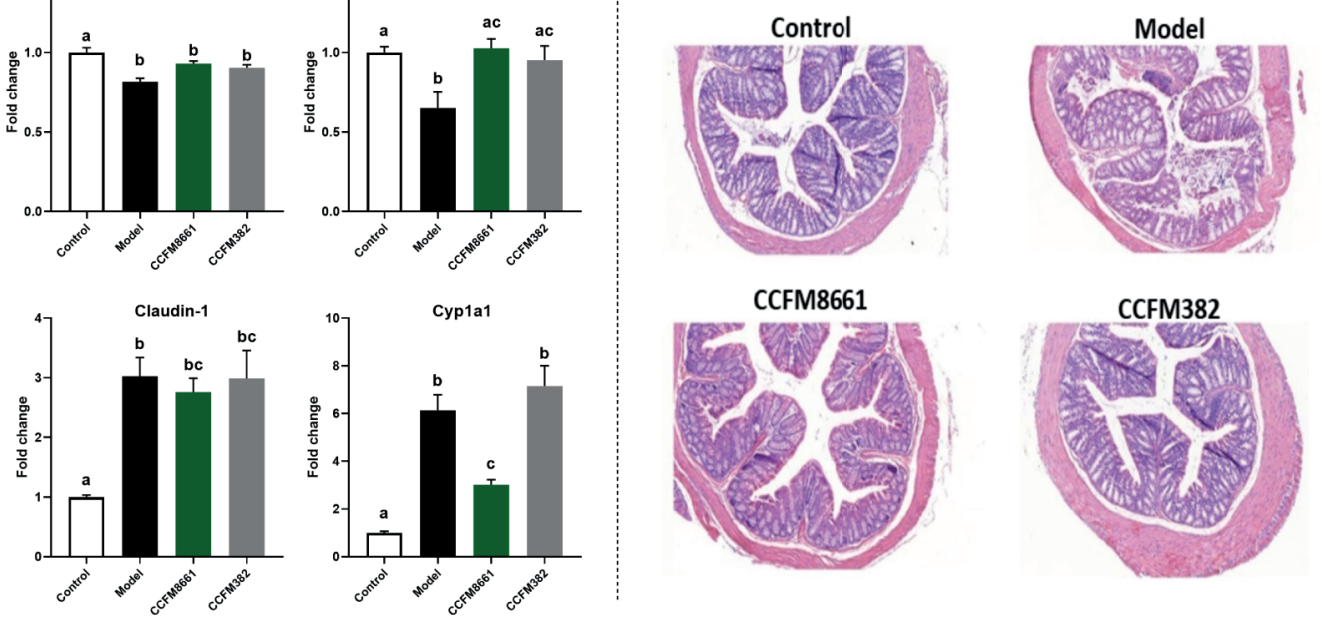

CCFM8661

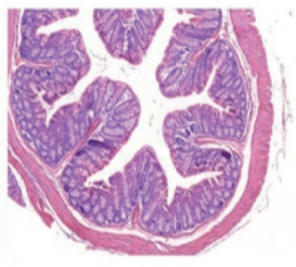

CCFM382

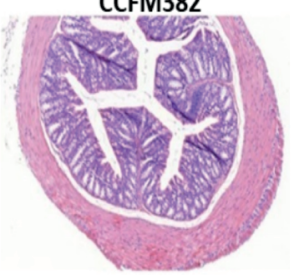

FIGURE 5 | Effect of $L$. plantarum CCFM8661 on the parameters related to the colon. (A) The mRNA expression of TJ proteins and CYP1A1. (B) Histopathological changes. The different letters indicate significant difference between groups $(P<0.05)$.

oral administration of $L$. plantarum CCFM8661 had a better alleviative effects on colonic damage induced by $\mathrm{B}[\alpha] \mathrm{P}$.

\section{Gut Microbiota Diversity}

To determine whether the protective effects of $L$. plantarum CCFM8661 on $\mathrm{B}[\alpha] \mathrm{P}$-induced colonic damage involved changes in the gut microbiota, the $\alpha$ diversity and composition of gut microbiota were measured. The number of observed species and the Shannon index were used to represent the gut microbiota richness and diversity, respectively. The Faith_pd index represents phylogenetic diversity, which is a qualitative measure of community richness. As shown in Figure 6A, the observed 
A
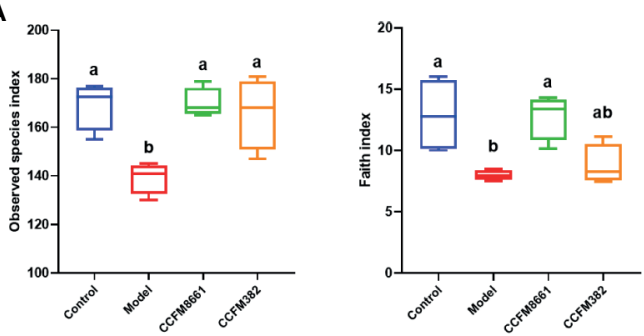

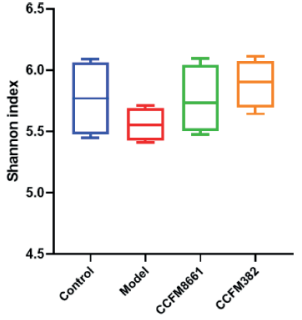

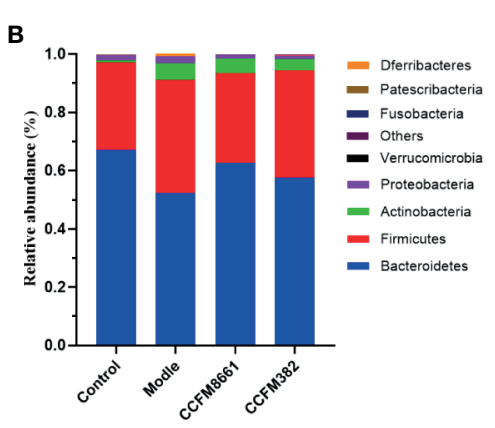
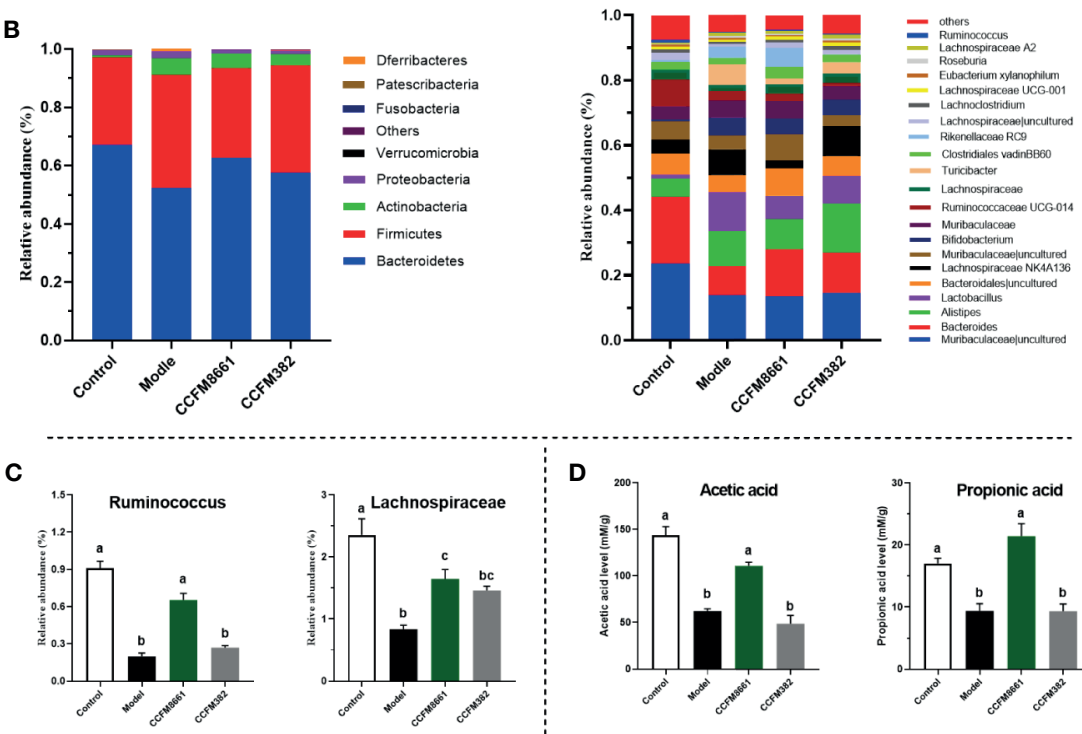

D
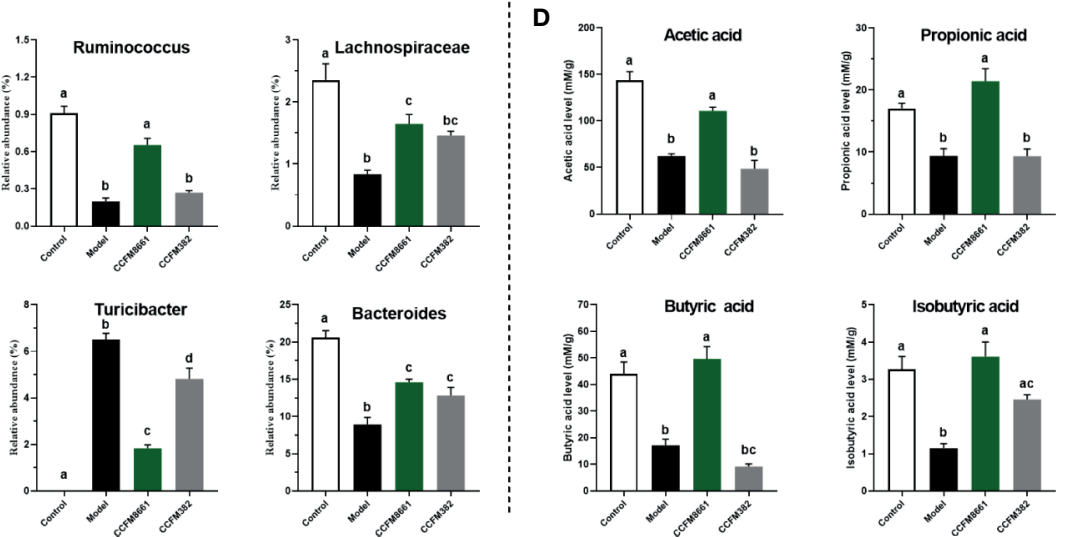

FIGURE 6 | Effect of $L$. plantarum CCFM8661 on the diversity and composition of the gut microbiota and SCFA levels. (A) $\alpha$-diversity, (B) relative abundance of constituents of the gut microbiota at the phylum and genus levels, (C) relative abundance of significant bacterial communities, (D) SCFA levels. The different letters indicate significant difference between groups $(P<0.05)$.

species and Faith_pd indices decreased significantly in the model group, indicating that the richness and diversity of the gut microbiota were dramatically reduced $(P<0.05)$. Oral supplementation of $L$. plantarum CCFM8661 significantly increased these two indexes, but L. plantarum CCFM382 supplementation only elevated the observed species index $(P<0.05)$. The results showed that L. plantarum CCFM8661 had a stronger regulating ability than CCFM382 on gut microbiota diversity.

\section{Composition of the Gut Microbiota}

The $\alpha$ diversity results confirmed that $\mathrm{B}[\alpha] \mathrm{P}$ and $L$. plantarum CCFM8661 could, indeed, change the composition of the intestinal microbiota. Thus, changes in the gut microbiota composition were further explored (Figure 6). At the phylum level, Bacteroidetes, Firmicutes, Actinobacteria, and Proteobacteria accounted for more than $95 \%$ of the gut microbiota (Figure 6B). In the control group, these four predominant bacterial phyla accounted for $67.1 \%, 30.1 \%, 0.7 \%$, and $1.8 \%$ of the gut microbiota, respectively. The abundance of Bacteroidetes, Firmicutes, and Actinobacteria in the model group was $52.2 \%, 38.9 \%$, and $5.8 \%$, respectively, whereas the abundance of Proteobacteria (2.3\%) remained almost unchanged. After supplement with $L$. plantarum CCFM8661, the abundance of Bacteroidetes (62.7\%), Firmicutes (30.8\%) and Proteobacteria (1.1\%) gradually recovered to the levels of the control group, while the abundance of Actinobacteria (5.0\%) was similar to their abundance in the model group, without significant changes. The abundances of these four bacterial phyla in CCFM382 
group accounted for $57.6 \%, 36.8 \%, 3.9 \%$ and $1.1 \%$ respectively. The results showed that CCFM382 also affected the gut microbiota composition, but the effects were not as strong as that of CCFM8661. At the genus level, Muribaculaceae, Bacteroides, Alistipes, and Lachnospiraceae NK4A136 accounted for $23.6 \%, 20.6 \%, 5.6 \%$, and $4.4 \%$, respectively, in the control group (Figure 6B). The abundance of Muribaculaceae and Bacteroides in the model group decreased to $13.8 \%$ and $8.9 \%$, respectively, and the abundance of Alistipes and Lachnospiraceae NK4A136 increased to $10.8 \%$ and $7.9 \%$, respectively, compared with those in the control group. In the CCFM8661 group, the abundance of Bacteroides increased to $14.6 \%$ and the abundance of Lachnospiraceae NK4A136 decreased to $2.5 \%$. Moreover, after $\mathrm{B}[\alpha] \mathrm{P}$ treatment, the abundance of Ruminococcus, Lachnospiraceae, and Bacteroides decreased significantly and the abundance of Turicibacter increased (Figure 6C, $P<0.05$ ). L. plantarum CCFM8661 reversed the abundance of these four genera to their levels observed in the control group $(P<0.05)$, while L. plantarum CCFM382 only reversed the abundances of Turicibacter and Bacteroides.

\section{SCFA Levels}

Compared to the levels in the control group, the levels of SCFAs, including acetic acid, butyric acid, isobutyric acid and propionic acid, were dramatically reduced in mice treated with $\mathrm{B}[\alpha] \mathrm{P}$ (Figure 6D). L. plantarum CCFM8661 sharply increased the levels of these SCFAs $(P<0.05)$ to levels almost the same as those in the control group. However, the administration of CCFM382 significantly increased only the levels of isobutyric acid among the SCFAs in $\mathrm{B}[\alpha] \mathrm{P}$-treated mice $(P<0.05)$. The results showed that L. plantarum CCFM8661 had a better effect than CCFM382 on increase of SCFA levels.

\section{Correlation and Heat Map Analyses}

The parameters that were significantly affected by $\mathrm{B}[\mathrm{a}] \mathrm{P}$ and L. plantarum CCFM8661 were selected to assess their correlation using Pearson's correlation coefficients (Figure 7). The Zo-1 expression levels were positive correlated with the abundance of Ruminococcus $(\mathrm{r}=0.91)$, Lachnospiraceae $(\mathrm{r}=0.99)$, and Bacteroides ( $\mathrm{r}=0.98)$, and negatively correlated with the Turicibacter abundance $(\mathrm{r}=-0.95)$, indicating that the gut microbiota was closely related to gut barrier function. The $3-\mathrm{OH} \mathrm{B}[\mathrm{a}] \mathrm{P}$ levels in feces had a strong positive correlation with Turicibacter abundance ( $\mathrm{r}=0.97)$ but a negative correlation with the abundance of Ruminococcus ( $\mathrm{r}=-0.97)$, Lachnospiraceae $(\mathrm{r}=-0.98)$, and Bacteroides $(\mathrm{r}=-0.99)$ and $\mathrm{Zo}-1$ expression levels $(\mathrm{r}=-0.96)$. These results confirmed that the gut microbiota and the gut barrier affected 3-OH $\mathrm{B}[\mathrm{a}] \mathrm{P}$ excretion in the feces, and vice versa. Moreover, $\mathrm{Bcl}-2$ expression levels showed a strong positive correlation with the abundance of Turicibacter $(\mathrm{r}=0.95)$ but a negative correlation with the abundance of Lachnospiraceae $(\mathrm{r}=-0.94)$ and Bacteroides ( $\mathrm{r}=-0.91) . p 53$ levels were positively correlated with Turicibacter abundance $(\mathrm{r}=1.00)$ and negatively correlated with the abundance Ruminococcus ( $\mathrm{r}=-0.98)$, Lachnospiraceae ( $\mathrm{r}=-0.93$ ) and Bacteroides ( $\mathrm{r}=-0.93)$. MDA levels were positively correlated with Turicibacter abundance $(\mathrm{r}=0.97)$ and negatively correlated with the Ruminococcus abundance $(\mathrm{r}=-0.94)$, indicating that the gut microbiota was related to changes in tumor- and oxidative stress- related parameters in the brain.

Heatmap analysis was used to identify the similarities and differences among the four groups, as similar groups would cluster together in this analysis. As shown in Figure 8, the CCFM8661 treatment group was clustered with the control group, and the CCFM382 treatment group clustered with the model group, indicating that CCFM8661 had a strong protection
A

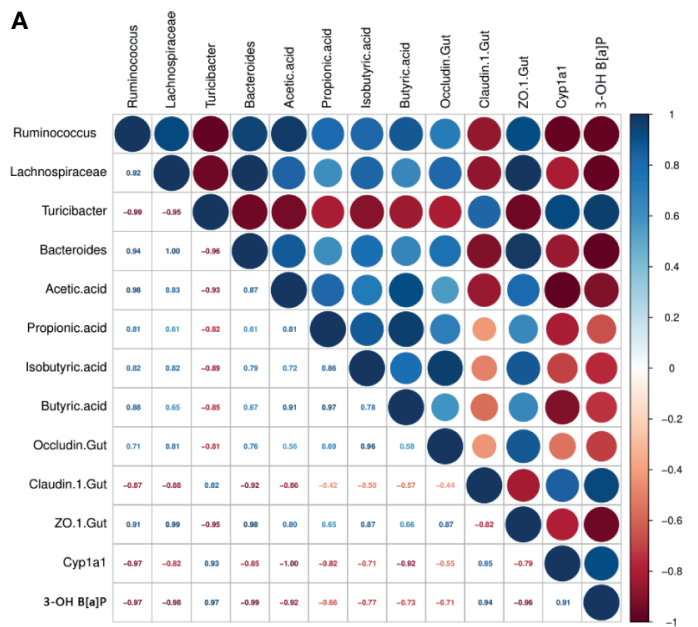

B

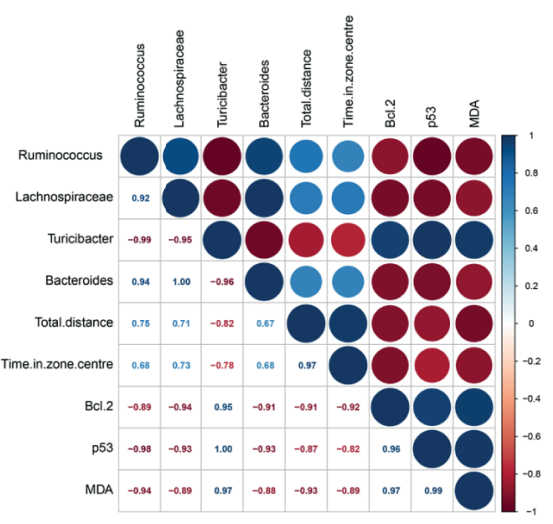

FIGURE 7 | Correlations between parameters significantly affected by B[a]P and L. plantarum CCFM8661 treatment. (A) The correlation of intestinal microbiota with gut-related parameters, (B) The correlation of intestinal microbiota with brain-related parameters. Significant negative and positive correlations are represented by red and blue circles, respectively. 


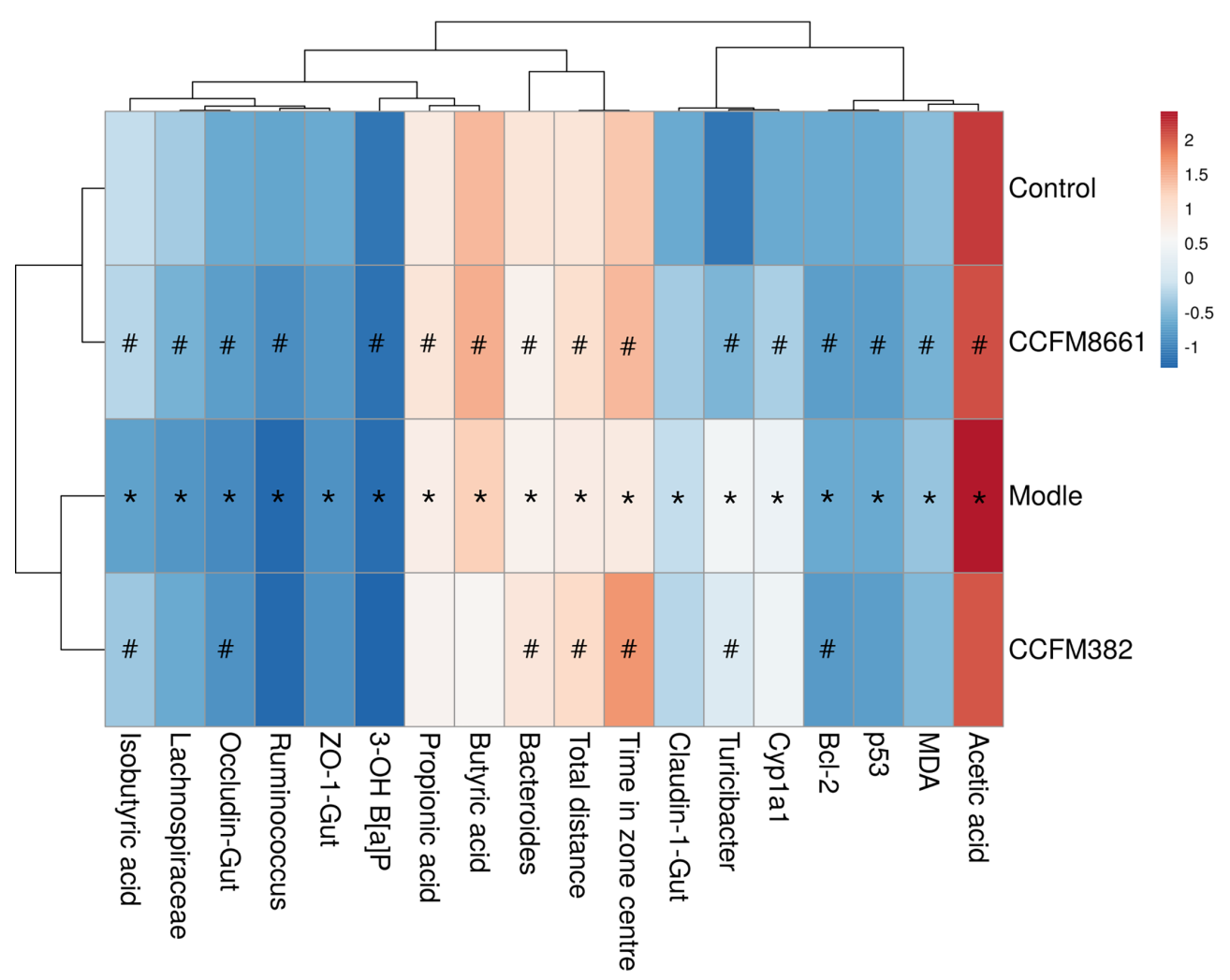

FIGURE 8 | Clustered heat map of the parameters significantly affected by B[a]P and L. plantarum CCFM8661 treatment. Red indicates an increase in the corresponding group and blue indicates a decrease in the corresponding group. The asterisks indicate significant difference between control and model groups $(P<$ 0.05). The pound signs indicate significant difference between model and $L$. plantarum intervention groups $(P<0.05)$.

against $\mathrm{B}[\mathrm{a}] \mathrm{P}$-induced damage, which may almost recover to the levels of the control group, while CCFM8661 only had a little protective effects.

\section{DISCUSSION}

When performing in vitro screening of strains of the probiotic L. plantarum for the potential to alleviate $\mathrm{B}[\alpha] \mathrm{P}$ toxicity, the $\mathrm{B}[\alpha] \mathrm{P}$-binding ability should be considered. The selected strain should have superb $\mathrm{B}[\alpha] \mathrm{P}$-binding ability, allowing it to bind $\mathrm{B}[\alpha] \mathrm{P}$ before it is absorbed by the host intestine, thereby resulting in excretion of $\mathrm{B}[\alpha] \mathrm{P}$ via the feces. In this study, the $\mathrm{B}[\alpha] \mathrm{P}$-binding capacities varied among the $23 \mathrm{~L}$. plantarum strains tested, and CCFM8661 was found to have the highest $\mathrm{B}[\alpha] \mathrm{P}$-binding ability. The adsorption of $\mathrm{B}[\alpha] \mathrm{P}$ and other carcinogens by Lactiplantibacillus strains has previously been reported, and the mechanisms may involve the adsorption of polysaccharides and proteins on the cell surface $(18,19,28)$. The main metabolic product of $\mathrm{B}[\alpha] \mathrm{P}$ in vivo is $3-\mathrm{OH} \mathrm{B}[\alpha] \mathrm{P}$. The levels of $3-\mathrm{OH} \mathrm{B}[\alpha] \mathrm{P}$ in the feces of mice treated with $\mathrm{B}[\alpha] \mathrm{P}$ were decreased after L. plantarum CCFM8661 supplementation, indicating that this strain may bind to $\mathrm{B}[\alpha] \mathrm{P}$ in vivo and, thereby, reduce its metabolism. It has been reported that probiotic
Lactiplantibacillus spp. can bind to heavy metals, such as lead (29) and cadmium (30), and mycotoxins, such as aflatoxin (31), in vivo, thereby alleviating their toxic effects.

$\mathrm{B}[\mathrm{a}] \mathrm{P}$ treatment disturbed the balance of the gut microbiota. At the phylum level, B[a]P elevated the abundance of Proteobacteria and Firmicutes and reduced the abundance of Bacteroidetes. It has been reported that an increase in Proteobacteria and a decrease in Bacteroidetes are associated with polyaromatic hydrocarbons (32, 33). At the genus level, the abundance of the beneficial bacteria Clostridiales, Lachnospiraceae, Ruminococcus, and Bacteroides decreased, and the abundance of the pro-inflammatory bacteria Turicibacter spp. increased significantly. However, L. plantarum CCFM8661 supplementation reversed these effects. A previous study also reported that $L$. salivarius Ls-33 can change the relative abundance of Clostridium spp. in the feces of obese juveniles (34). Some Clostridiales members produce butyrate, which may have an anti-inflammatory effect (13), and its levels usually decrease in an unhealthy state (35). Members of the family Lachnospiraceae are the most important butyrate-producing microorganisms in the intestine, as they regulate host energy metabolism and mucosal integrity and create a proinflammatory environment (36), while Ruminococcus has the ability to regulate mucin expression and mucosal glycosylation in the colonic mucosa (37). The significant changes observed in the abundance of these bacteria indicated that 
$\mathrm{B}[\mathrm{a}] \mathrm{P}$ treatment can disrupt the gut microbiota composition, and L. plantarum CCFM8661 intervention can improve the homeostasis of the intestinal microbiota.

SCFAs are the metabolites of the gut microbiota, which can directly provide energy for intestinal epithelial cells and affect intestinal immunity and barrier function $(38,39)$. In this study, B [a]P exposure led to a decrease in the expression levels of the TJrelated genes $\mathrm{Zo}-1$ and occludin; this decrease may be attributed to the decrease in butyric and isobutyric acid production caused by $\mathrm{B}[\mathrm{a}] \mathrm{P}$. L. plantarum CCFM8661 supplementation significantly increased the expression levels of occludin to almost normal levels. Previous studies have also reported that Lactiplantibacillus intervention can induce colonic TJ-related protein expression (40). In summary, B[a]P can significantly reduce the diversity of the intestinal microbiota, thus increasing the permeability of the intestinal barrier. Notably, L. plantarum CCFM8661 protected the integrity of the colonic mucosa and alleviated the pathological damage to the colon, which may have been related to changes in the intestinal microbiota. In addition, L. plantarum CCFM8661 significantly reduced the level of CYP1A1 induced by $\mathrm{B}[\mathrm{a}] \mathrm{P}$ and, thus, exerted a protective effect on the colon. Pithva et al. demonstrated similar results, showing that L. rhamnosus Vc alleviates colon injury caused by the carcinogen N-methyl-N '-nitroguanidine (41).

Mood and behavior are also closely related to changes in the intestine microbiota (42). B[a]P treatment resulted in disturbances of the gut microbiota, resulting in brain injuries and behavioral abnormalities. However, L. plantarum CCFM8661 reversed these effects. Bcl-2 has anti-apoptotic roles, and its overexpression can reduce the production of oxygen free radicals and lipid peroxides and inhibit changes in mitochondrial permeability, thus inhibiting apoptosis. Exposure to $\mathrm{B}[\mathrm{a}] \mathrm{P}$ and the pesticide triazophos has been shown to significantly increase $B c l-2$ expression levels in the brain (43). The tumor suppressor gene $p 53$ is one of the most frequently mutated genes and is highly correlated with human cancers. Abnormal expression of this gene can be found in more than $50 \%$ of all malignant tumors. When DNA damage is minor, the p53 gene helps the cell to repair itself; however, if the damage is severe or the DNA repair mechanism fails, p53 induces apoptosis. Intraperitoneal injection of $\mathrm{B}[\alpha] \mathrm{P}$ has been shown to increase the expression levels of $p 53$ in the cerebral cortex and hippocampus (44). Lactiplantibacillus administration has been shown to protect the brain from $\mathrm{B}[\alpha] \mathrm{P}$-induced injury by reducing the expression levels of $\mathrm{Bcl}-2$ and $\mathrm{p} 53$. In addition, an increase in intestinal permeability induces systemic inflammation, causing an inflammatory response in the brain. The levels of MDA, an oxidative stress factor, reflect lipid peroxidation levels, which indicate the extent of injury to cells (45). A previous study also showed that $\mathrm{B}[\alpha] \mathrm{P}$ exposure increases MDA levels in the brain (46), indicating ROS generation and brain injury (47). Reportedly, $L$. plantarum strains have antioxidant abilities and induce a decrease in MDA levels (48).

In present study, the protection of two L. plantarum strains, with the highest and lowest $\mathrm{B}[\mathrm{a}] \mathrm{P}$-binding ability respectively, against $\mathrm{B}[\mathrm{a}] \mathrm{P}$-induced toxicity were compared. In terms of decreasing 3-OH $\mathrm{B}[\mathrm{a}] \mathrm{P}$ level in feces, improving colonic histopathology, oxidative stress- and tumor-related parameters in the brain and behavioral performance, regulating diversity and composition of gut microbiota and increasing SCFAs level, L. plantarum CCFM8661 performed better than L. plantarum CCFM382. The results of Heatmap analysis also proved this viewpoint. The underlying reasons for these different effects may main involve their significant difference in $\mathrm{B}[\mathrm{a}] \mathrm{P}$-binding ability. However, in the aspect of colonic TJs expression and gut microbiota diversity, L. plantarum CCFM382 and L. plantarum CCFM8661 had the similar performance, which may attribute to the antioxidative ability or other probiotic properties of L. plantarum CCFM382.

\section{CONCLUSION}

Oral administration of $L$. plantarum CCFM8661 effectively alleviated colonic histopathological changes, reduced oxidative stress and tumor-related parameters in the brain, and improved behavioral performance. The underlying mechanism for these effects may involve the B[a]P-binding ability of CCFM8661 or

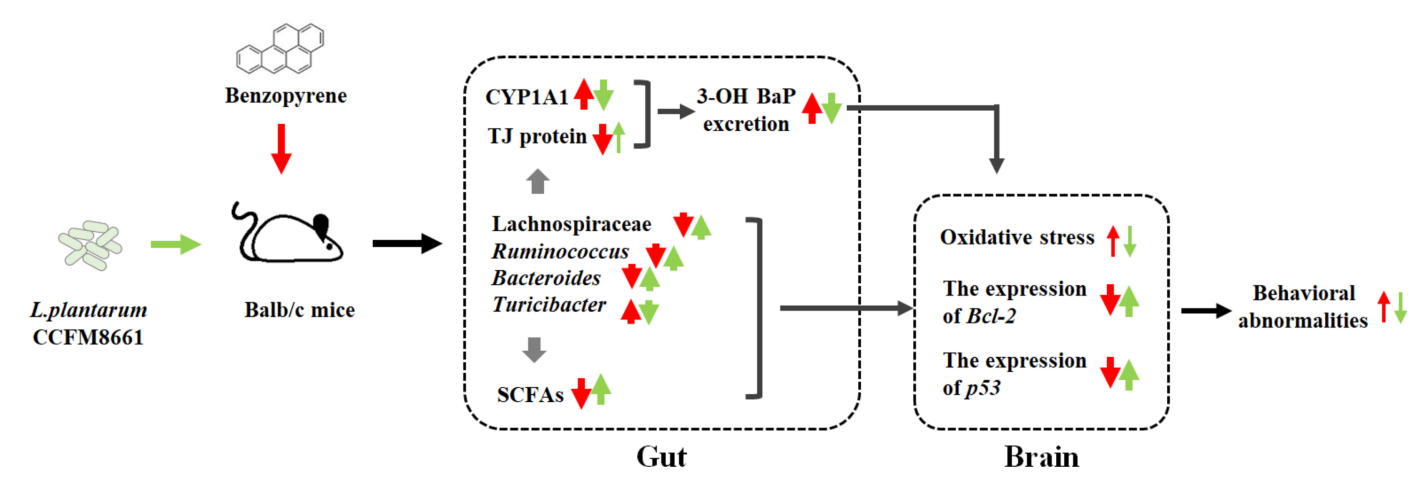

FIGURE 9 | The possible protective mechanisms of $L$. plantarum CCFM8661 against B[a]P toxicity in the gut and brain. The red and green arrows represent the $\mathrm{B}[\mathrm{a}] \mathrm{P}$ - and CCFM8661-induced parameter changes, respectively. The thin arrows represent that only part of the parameter is affected. 
the regulation of the gut microbiota by CCFM8661, including an increase in the gut microbiota diversity and the abundance of Ruminococcus and Lachnospiraceae and a decrease in the abundance of the pro-inflammatory Turicibacter spp., thereby increasing the SCFA levels and improving the integrity of the gut barrier (Figure 9). Therefore, oral administration of L. plantarum CCFM8661 is an effective, easy, and safe intervention against $\mathrm{B}[\mathrm{a}] \mathrm{P}$-induced toxicity.

\section{DATA AVAILABILITY STATEMENT}

The data presented in the study are deposited in the NCBI repository, accession number from SRR15244744 to SRR15244788.

\section{ETHICS STATEMENT}

All procedures and protocols of mice experiments were performed according to the guidelines of the Animal Care and Use Committee and the Ethics Committee of Jiangnan University (JN.No20190915b0481210).

\section{REFERENCES}

1. Boström CE, Gerde P, Hanberg A, Jernström B, Johansson C, Kyrklund T, et al. Cancer Risk Assessment, Indicators, and Guidelines for Polycyclic Aromatic Hydrocarbons in the Ambient Air. Environ Health Persp (2002) 110:451-88. doi: 10.1289/ehp.110-1241197

2. Jeong KH, Lee HJ, Park T, Shim SM. Catechins Controlled Bioavailability of Benzo a Pyrene (B Alpha P) From the Gastrointestinal Tract to the Brain Towards Reducing Brain Toxicity Using the In Vitro Bio-Mimic System Coupled With Sequential Co-Cultures. Molecules (2019) 24(11):2175-5. doi: 10.3390/molecules24112175

3. Lee BM, Shim GA. Dietary Exposure Estimation of Benzo[a]Pyrene and Cancer Risk Assessment. J Toxicol Env Heal A (2007) 70(15-16):1391-4. doi: $10.1080 / 15287390701434182$

4. Stumpe-Viksna I, Morozovs A, Bartkevics V, Kukāre A. Levels of Benzo(a) Pyrene (BaP) in Fish, Smoked According to Different Procedures. Proc Latvia Univ Agric (2008) 21(315):24-9.

5. Omidian K, Rafiei H, Bandy B. Polyphenol Inhibition of Benzo[a]PyreneInduced Oxidative Stress and Neoplastic Transformation in an In Vitro Model of Carcinogenesis. Food Chem Toxicol (2017) 107:523-5. doi: 10.1016/ j.fct.2017.07.028

6. Chepelev NL, Moffat ID, Bowers WJ, Yauk CL. Neurotoxicity may be an Overlooked Consequence of Benzo[a]Pyrene Exposure That Is Relevant to Human Health Risk Assessment. Mutat Res-Rev Mutat (2015) 764:64-89. doi: 10.1016/j.mrrev.2015.03.001

7. Irmawati A, Jasmin N, Sidarningsih. The Effect of Moderate Exercise on the Elevation of Bax/Bcl-2 Ratio in Oral Squamous Epithelial Cells Induced by Benzopyrene. Vet World (2018) 11(2):177-80. doi: 10.14202/ vetworld.2018.177-180

8. Yu D, Kazanietz MG, Harvey RG, Penning TM. Polycyclic Aromatic Hydrocarbon O-Quinones Inhibit the Activity of the Catalytic Fragment of Protein Kinase C. Biochemistry (2002) 41(39):11888-94. doi: 10.1021/ bi020270p

9. Grova N, Valley A, Turner JD, Morel A, Schroeder H. Modulation of Behavior and NMDA-R1 Gene mRNA Expression in Adult Female Mice After SubAcute Administration of Benzo(a)Pyrene. Neurotoxicology (2007) 28(3):6306. doi: 10.1016/j.neuro.2007.01.010

10. Perera FP, Rauh V, Tsai WY, Kinney P, Camann D, Barr D, et al. Effects of Transplacental Exposure to Environmental Pollutants on Birth Outcomes in a

\section{AUTHOR CONTRIBUTIONS}

LY: Methodology, Software, Formal analysis, Visualization, Funding acquisition, Writing—original draft. LZ: Investigation, Methodology, Software. HD: Writing-original draft, Methodology, Formal analysis. RZ: Investigation, Formal analysis. YX: Software, Software. MG: Investigation, Methodology. JZ: Supervision, Validation. HZ: Supervision, Validation. WC: Project administration, Funding acquisition. FT: Conceptualization, Writing-review and editing, Funding acquisition. All authors contributed to the article and approved the submitted version.

\section{FUNDING}

This work was supported by National Natural Science Foundation of China Key Program (31772090, 32001665, 31820103010, U1903205), Natural Science Foundation of Jiangsu Province (BK20180603), the Key Scientific and Technological Research Projects (2018AB010), BBSRC Newton Fund Joint Centre Award, the National first-class discipline program of Food Science and Technology (JUFSTR20180102).

Multiethnic Population. Environ Health Perspect (2003) 111(2):201-5. doi: 10.1289/ehp. 5742

11. Laurent C, Feidt C, Grova N, Mpassi D, Rychen G. Portal Absorption of 14C After Ingestion of Spiked Milk With 14C-Phenanthrene, 14C-Benzo[a]Pyrene or 14C-TCDD in Growing Pigs. Chemosphere (2008) 48(8):843-8. doi: 10.1016/S0045-6535(02)00145-5

12. Wakx AS, Regazzetti A, Dargère D, Auzeil N, Gil S, Brion DE. New In Vitro Biomarkers to Detect Toxicity in Human Placental Cells: The Example of Benzo[A]pyrene. Toxicol Vitro (2016) 32:76-85. doi: 10.1016/j.tiv.2015.11.022

13. Ribière C, Peyret P, Parisot N, Darcha C, Déchelotte PJ, Barnich N, et al. Oral Exposure to Environmental Pollutant Benzo[a]Pyrene Impacts the Intestinal Epithelium and Induces Gut Microbial Shifts in Murine Model. Sci Rep (2016) 6(1):31027. doi: 10.1038/srep31027

14. Li M, Esch BC, Wagenaar GT, Garssen J, Folkerts G, Henricks P. Pro-And Anti-Inflammatory Effects of Short Chain Fatty Acids on Immune and Endothelial Cells. Eur J Pharmacol (2018) 831:52-9. doi: 10.1016/ j.ejphar.2018.05.003

15. Vadder FD, Kovatcheva-Datchary P, Goncalves D, Vinera J, Mithieux G. Microbiota-Generated Metabolites Promote Metabolic Benefits via GutBrain Neural Circuits. Cell (2014) 156(1-2):84-96. doi: 10.1016/j.cell.2013. 12.016

16. Koh A, Vadder FD, Kovatcheva-Datchary P, Bäckhed F. From Dietary Fiber to Host Physiology: Short-Chain Fatty Acids as Key Bacterial Metabolites. Cell (2016) 165(6):1332-45. doi: 10.1016/j.cell.2016.05.041

17. Kimoto-Nira H. New Lactic Acid Bacteria for Skin Health via Oral Intake of Heat-Killed or Live Cells. Ani Sci J (2018) 89(6):835-42. doi: 10.1111/ asj. 13017

18. Suzuki T, Yamazaki K, Shinoda T, Shirai M, Yoshikawa H, Noguchi Y, et al. Excretion of 3,3',4,4', 5-Pentachlorobiphenyl (PCB126) From Rat Liver Following Oral Administration of Lactobacillus Reuteri and Lactobacillus Acidophilus[J]. Food Sci Technol Res (2014) 20(4):821-8. doi: 10.3136/ fstr.20.821

19. Apás AL, González SN, Arena ME. Potential of Goat Probiotic to Bind Mutagens[J]. Anaerobe (2014) 28:8-12. doi: 10.1016/j.anaerobe.2014.04.004

20. Liu Y, Wu J, Xiao Y, Liu Q, Zhai Q. Relief of Cadmium-Induced Intestinal Motility Disorder in Mice by Lactobacillus Plantarum CCFM8610. Front Immunol (2020) 11:619574. doi: 10.3389/fimmu.2020.619574

21. Zhao H, Zhou F, Qi Y, Dziugan P, Bai F, Walczak P, et al. Screening of Lactobacillus Strains for Their Ability to Bind Benzo (a) Pyrene and the 
Mechanism of the Process. Food Chem Toxicol (2013) 59:67-71. doi: 10.1016/ j.fct.2013.05.040

22. Burokas A, Arboleya S, Moloney RD, Peterson VL, Murphy K, Clarke G, et al. Targeting the Microbiota-Gut-Brain Axis: Prebiotics Have Anxiolytic and Antidepressant-Like Effects and Reverse the Impact of Chronic Stress in Mice. Biol Psychiatry (2017) 82(7):472-87. doi: 10.1016/j.biopsych.2016. 12.031

23. Cryan JF, Markou A, Lucki I. Assessing Antidepressant Activity in Rodents: Recent Developments and Future Needs. Trends Pharmacol Sci (2002) 23 (5):238-45. doi: 10.1016/S0165-6147(02)02017-5

24. Liu X, Qi L, Yang B, Su D, Li F, Liu H. Determination of Benzo (a)Pyrene and 3-Hydroxy Benzo(a)Pyrene From Shellfish in Dalian. Chin J Health Lab Technol (2013) 23(5):1234-44.

25. Xu Q, Li X, Wang E, He Y, Yin B, Fang D, et al. A Cellular Model for Screening of Lactobacilli That Can Enhance Tight Junctions. RSC Adv (2016) 6 (113):111812-21. doi: 10.1039/C6RA24148D

26. Yu L, Zhai Q, Zhu J, Zhang C, Li T, Liu X, et al. Dietary Lactobacillus Plantarum Supplementation Enhances Growth Performance and Alleviates Aluminum Toxicity in Tilapia. Ecotoxicol Environ Saf (2017) 143:307-14. doi: 10.1016/j.ecoenv.2017.05.023

27. Wang G, Li X, Zhao J, Hao Z, Wei C. Lactobacillus Casei CCFM419 Attenuates Type 2 Diabetes via a Gut Microbiota Dependent Mechanism. Food Funct (2017) 8(9):3155-64. doi: 10.1039/C7FO00593H

28. Lin D, Ji R, Wang D, Li Y, Qin T, Xing B, et al. The Research Progress in Mechanism and Influence of Biosorption Between Lactic Acid Bacteria and $\mathrm{Pb}$ (II): A Review. Crit Rev Food Sci Nutr (2019) 59(3):395-410. doi: 10.1080/ 10408398.2017.1374241

29. Zafarullah M, Rabia R, Zhang S, Hu H, Ahsan H, Bakry AM, et al. Comparative Assessment of the Bioremedial Potentials of Potato Resistant Starch-Based Microencapsulated and Non-Encapsulated Lactobacillus Plantarum to Alleviate the Effects of Chronic Lead Toxicity. Front Microbiol (2018) 9:1306. doi: 10.3389/fmicb.2018.01306

30. Zhai Q, Wang G, Zhao J, Liu X, Narbad A. Protective Effects of Lactobacillus Plantarum CCFM8610 Against Chronic Cadmium Toxicity in Mice Indicate Routes of Protection Besides Intestinal Sequestration. Appl Environ Microb (2014) 80(13):4063-71. doi: 10.1128/AEM.00762-14

31. Zhang LY, Liu S, Zhao XJ, Wang N, Jiang X, Xin HS, et al. Lactobacillus Rhamnosus GG Modulates Gastrointestinal Absorption, Excretion Patterns, and Toxicity in Holstein Calves Fed a Single Dose of Aflatoxin B1. J Dairy Sci (2019) 102(2):1330-40. doi: 10.3168/jds.2018-15444

32. Mukherjee S, Juottonen H, Siivonen P, Quesada CL, Tuomi P, Pulkkinen P, et al. Spatial Patterns of Microbial Diversity and Activity in an Aged Creosote-Contaminated Site. Isme J (2014) 8(10):2131-42. doi: 10.1038/ ismej.2014.151

33. Parajuli A, Gronroos M, Kauppi S, Plociniczak T, Roslund M, Galitskaya P, et al. The Abundance of Health-Associated Bacteria is Altered in PAH Polluted Soils-Implications for Health in Urban Areas? PLoS One (2017) 12 (11):e0187852. doi: 10.1371/journal.pone.0187852

34. Larsen N, Vogensen FK, Gobel RJ, Michaelsen KF, Jakobsen M, et al. Effect of Lactobacillus Salivarius Ls-33 on Fecal Microbiota in Obese Adolescents. Clin Nutr (2013) 32(6):935-40. doi: 10.1016/j.clnu.2013.02.007

35. Gevers D, Kugathasan S, Denson LA, Baeza YV, Treuren WV, Ren B, et al. The Treatment-Naive Microbiome in New-Onset Crohn's Disease. Cell Host Microbe (2014) 15(3):382-92. doi: 10.1016/j.chom.2014.02.005

36. Zhang J, Song L, Wang Y, Liu C, Zhang L, Zhu S, et al. Reduction in ButyrateProducing Lachnospiraceae Contributes to Stress-Induced Visceral Hypersensitivity in Rats. J Gastroen Hepatol (2019) 34(8):1368-76. doi: 10.1111/jgh.14536
37. Graziani F, Pujol A, Nicoletti C, Dou S, Maresca M, Giardina T, et al. Ruminococcus Gnavus E1 Modulates Mucin Expression and Intestinal Glycosylation. J Appl Microbiol (2016) 120(5):1403-17. doi: 10.1111/ jam.13095

38. Rooks MG, Garrett WS. Gut Microbiota, Metabolites and Host Immunity. Nat Rev Immunol (2016) 16(6):341-52. doi: 10.1038/nri.2016.42

39. Ploeger S, Stumpff F, Penner GB, Schulzke JD, Gäbel G, Martens H, et al. Aschenbach: Microbial Butyrate and its Role for Barrier Function in the Gastrointestinal Tract. Ann N Y Acad Sci (2012) 1258:52-9. doi: 10.1111/ j.1749-6632.2012.06553.x

40. Jeong JJ, Kim KA, Hwang YJ, Han MJ, Kim DH. Anti-Inflammaging Effects of Lactobacillus Brevis OW38 in Aged Mice. Benef Microbe (2016) 7(5):707-18. doi: 10.3920/BM2016.0016

41. Pithva SP, Ambalam PS, Ramoliya JM, Dave JM, Vyas BRM. Antigenotoxic and Antimutagenic Activities of Probiotic Lactobacillus Rhamnosus Vc Against N-Methyl-N'-Nitro-N-Nitrosoguanidine. Nutr Cancer (2015) 67 (7):1142-50. doi: 10.1080/01635581.2015.1073751

42. Dinan TG, Cryan JF. Gut-brain Axis in 2016: Brain-Gut-Microbiota Axis Mood, Metabolism and Behaviour. Nat Rev Gastro Hepat (2017) 14(2):69-70. doi: $10.1038 /$ nrgastro.2016.200

43. Wang G, Shao J, Wu M, Meng Y, Gul Y, Yang H, et al. Effect of Acute Exposure of Triazophos on Histological Structure and Apoptosis of the Brain and Liver of Zebrafish (Danio Rerio). Ecotox Environ Safe (2019) 180:646-55. doi: 10.1016/j.ecoenv.2019.05.053

44. He J, Ji X, Li Y, Xue X, Feng G, Zhang H, et al. Subchronic Exposure of Benzo (a)Pyrene Interferes With the Expression of Bcl-2, Ki-67, C-Myc and P53, Bax, Caspase-3 in Sub-Regions of Cerebral Cortex and Hippocampus. Exp Toxicol Pathol (2016) 68(2-3):149-56. doi: 10.1016/j.etp.2015.11.007

45. Apel K, Hirt H. Reactive Oxygen Species: Metabolism, Oxidative Stress, and Signal Transduction. Annu Rev Plant Biol (2004) 55:373-99. doi: 10.1146/ annurev.arplant.55.031903.141701

46. Saunders CR, Das SK, Ramesh A, Shockley DC, Mukherjee S. Benzo(a) pyrene-Induced Acute Neurotoxicity in the F-344 Rat: Role of Oxidative Stress. J Appl Toxicol (2006) 26(5):427-38. doi: 10.1002/jat.1157

47. Dutta K, Ghosh D, Nazmi A, Kumawat KL, Basu A. A Common Carcinogen Benzo[a]pyrene Causes Neuronal Death in Mouse via Microglial Activation. PloS One (2010) 5(4):e9984 50. doi: 10.1371/journal.pone.0009984

48. Yu L, Zhai Q, Liu X, Wang G, Zhang Q, Zhao J, et al. Lactobacillus Plantarum CCFM639 Alleviates Aluminium Toxicity. Appl Microbiol Biotechnol (2016) 100(4):1891-900. doi: 10.1007/s00253-015-7135-7

Conflict of Interest: The authors declare that the research was conducted in the absence of any commercial or financial relationships that could be construed as a potential conflict of interest.

Publisher's Note: All claims expressed in this article are solely those of the authors and do not necessarily represent those of their affiliated organizations, or those of the publisher, the editors and the reviewers. Any product that may be evaluated in this article, or claim that may be made by its manufacturer, is not guaranteed or endorsed by the publisher.

Copyright $\odot 2021$ Yu, Zhang, Duan, Zhao, Xiao, Guo, Zhao, Zhang, Chen and Tian. This is an open-access article distributed under the terms of the Creative Commons Attribution License (CC BY). The use, distribution or reproduction in other forums is permitted, provided the original author $(s)$ and the copyright owner(s) are credited and that the original publication in this journal is cited, in accordance with accepted academic practice. No use, distribution or reproduction is permitted which does not comply with these terms. 